\title{
How to Triage the Red Eye: Considerations for Non-ophthalmic Providers
}

\section{Braich $\mathrm{PS}^{{ }^{*}}$ and Kuriakose $\mathbf{R}^{2}$}

${ }^{1}$ Department of Ophthalmology, Virginia Commonwealth University School of Medicine, Richmond, VA, USA

${ }^{2}$ Virginia Commonwealth University School of Medicine, Richmond, VA, USA

Keywords: Red eye; Eye emergency; Triaging a red eye

\section{Introduction}

This short communication is intended for nurses and other midlevel non-physician providers to triage which patients with a red eye need to be evaluated by an eye care provider urgently. This brief review on how to triage a red eye is in no way a complete assessment of all of the potential etiologies, therefore it should never replace consultation with your eye care provider.

One of the most helpful differentiating symptoms of a red eye, also called injection, is pain. In general, the non-painful red eye denotes a less serious, non-vision threatening cause [1,2]. A painful red eye is often a more serious problem. Furthermore, patients who wear contact lenses and have a red eye with or without pain should also be considered high risk patients and warrant urgent evaluation.

When presenting information to an eye care provider a few pieces of information are essential in triaging patients. These exam findings, often referred to as the "ophthalmic vital signs," are vision, intraocular pressure (IOP) and pupils. Vision can be checked with a distance chart (20 feet) or near card for emergency purposes but should always be checked one eye at a time and with the patient's glasses on if they have them. For the non-ophthalmic provider who may be untrained in performing applanation tonometry, the intraocular pressure in emergency situations can be checked by a tono-pen or at least by digital palpation with the thumb to gauge what is soft versus hard. The fellow eye or the examiner's eye can be used as a reference.

Slit lamp examination, by which more than $90 \%$ of red eyes can be diagnosed, is extremely helpful and if available should be used. This communication presents a few photos for reference purposes, but readers are encouraged to look at a photo-atlas of similar pathology as ophthalmic diseases follow certain visual patterns and often the diagnosis is apparent just after history and physical examination alone (Figure 1). All photos of clinical pathology were selected from the Kanski Clinical Ophthalmology Review Book [1].

\section{Painful Red Eye}

Proptosis, conjunctival injection, limited ocular motility and pain are highly suggestive of inflammatory orbital diseases such as orbital pseudotumor (Figure 2) or orbital cellulitis (Figure 3). The latter is an ophthalmic emergency and suspicion of it warrants ophthalmic consultation for possible hospital admission and intravenous antibiotics.

Pain, diffuse conjunctival injection, high intraocular pressure (IOP), mid-dilated pupil, in a patient who is nauseous or may be vomiting are suggestive of acute angle closure. If a tono-pen is not available then gentle palpation with the thumb comparing the affected eye to a normal eye will be very useful information in gauging the IOP. This condition warrants emergent eye care.

Pain, diffuse scleral injection, and tenderness to palpation suggest a diagnosis of scleritis. This requires a systemic workup based on a thorough review of systems to rule out both generalized immune diseases and infectious causes. Discerning between scleritis and episcleritis may be challenging to the beginner and ophthalmic consultation is advised prior to work up and treatment.

A helpful tool to have when evaluating certain corneal pathology in patients with a red eye is fluorescein staining and a cobalt blue light. A popular alternative is the Woods light. The cornea is very highly innervated with sensory fibers and in many cases sloughing of the corneal epithelium results in intense pain. The provider may be urged to provide topical anesthesia as a treatment. Although this brings the patient comfort and helps in diagnosis, it is not therapeutic. Chronic use of topical anesthetics can lead to serious problems for ocular health and should be avoided. The differential diagnosis of the positive corneal staining includes corneal abrasion (Figure 4), foreign body (Figure 5), herpes simplex keratitis, keratoconjunctivitis or corneal ulcers to list just a few.

The red eye, with photophobia and anterior chamber inflammation, which requires a slit lamp to view, may suggest uveitis. An easy diagnostic maneuver to determine the presence of anterior uveitis is to test whether the patient has pain when shining a light into the affected eye and the unaffected eye. The term for this is consensual photophobia and due to the cross over innervation of the pupils, is highly specific for anterior uveitis. Patients may also exhibit posterior chamber inflammation in the form of vitritis, retinitis, vasculitis, or infective endophthalmitis. Slit lamp exam and indirect ophthalmoscopy are needed to determine the presence of these other findings of uveitis.

In the red eye with pain and ciliary injection, the presence of hypopyon (a layer of white blood cells in the anterior chamber) points towards a diagnosis which can be infectious, autoimmune or malignant (Figure 6).

Hyphema (a layer of red blood cells in the anterior chamber) after trauma makes the diagnosis of a painful red eye apparent (Figure 7). This is something the ophthalmic provider should be aware since there are a variety of ways these are managed and follow up would need to be maintained to ensure there is no rebleeding and intraocular pressure is controlled. When found these patients should have their affected eye shielded to avoid inadvertently poking their eye. They should be instructed to sit still and taken off of nonsteroidal anti-inflammatory medication.

*Corresponding author: Puneet S. Braich, Department of Ophthalmology Virginia Commonwealth University, $401 \mathrm{~N} \mathrm{11th} \mathrm{St,} \mathrm{Suite} \mathrm{439,} \mathrm{Nelson} \mathrm{Clinic} \mathrm{4th}$ floor, Richmond, VA 23298, USA, Tel: 8048288643; E-mail: Puneet.Braich@aya. yale.edu

Received December 12, 2015; Accepted February 24, 2016; Published March 02,2016

Citation: Braich PS, Kuriakose R (2016) How to Triage the Red Eye: Considerations for Non-Ophthalmic Providers. Adv Practice Nurs 2:105. doi:10.4172/25730347.1000105

Copyright: $\odot 2016$ Braich P, et al., This is an open-access article distributed under the terms of the Creative Commons Attribution License, which permits unrestricted use, distribution, and reproduction in any medium, provided the original author and source are credited. 
Citation: Braich PS, Kuriakose R (2016) How to Triage the Red Eye: Considerations for Non-ophthalmic Providers. Adv Practice Nurs 2:105. doi:10.4172/2573-0347.1000105

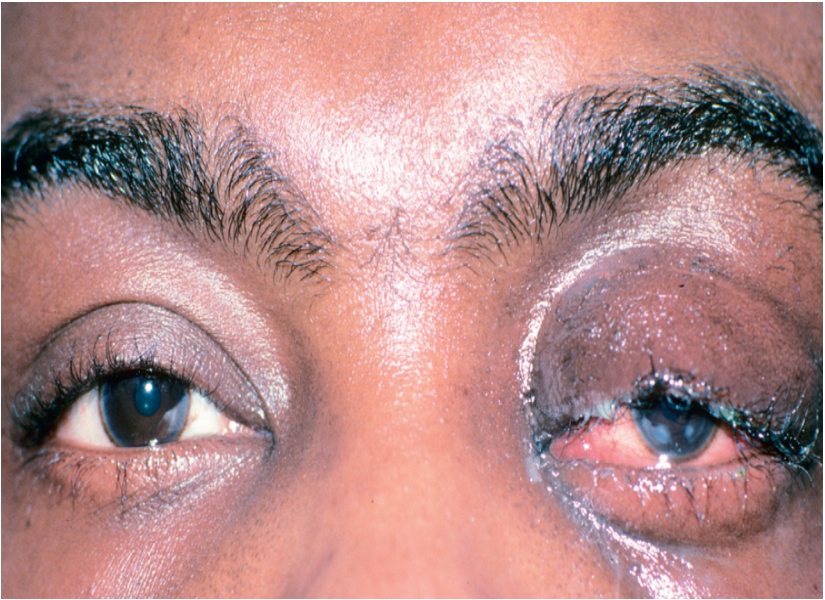

Figure 1: Orbital Pseudo tumor aka nonspecific orbital inflammation. Notice the proptosis, injection, and soft tissue swelling. Image courtesy of Kanski, JJ. Clinical ophthalmology: a systematic approach. Elsevier/Saunders; 2011.

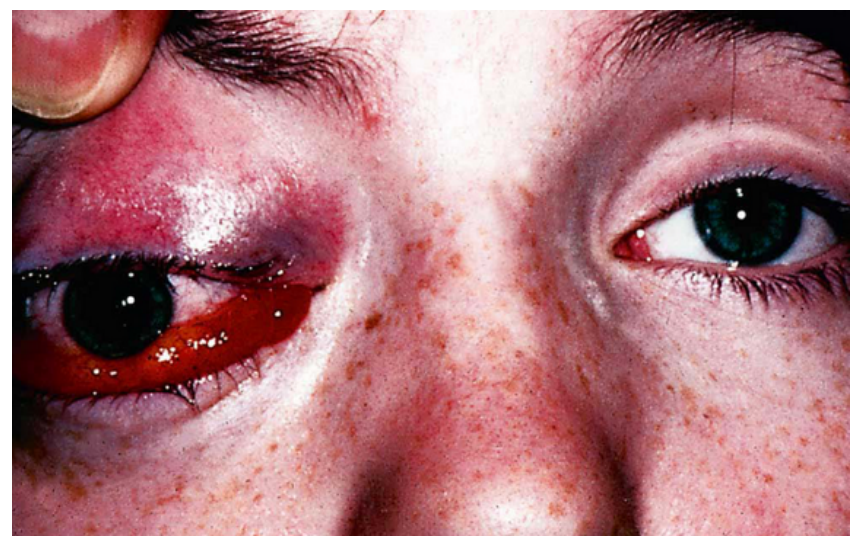

Figure 2: Orbital Cellulitis, which shows many signs of orbital inflammation This patient's right eye also demonstrates hypoglobus. Often orbital cellulitis and orbital pseudotumor can have identical signs and symptoms. Image courtesy of Kanski, JJ. Clinical ophthalmology: a systematic approach. Elsevier/Saunders; 2011.
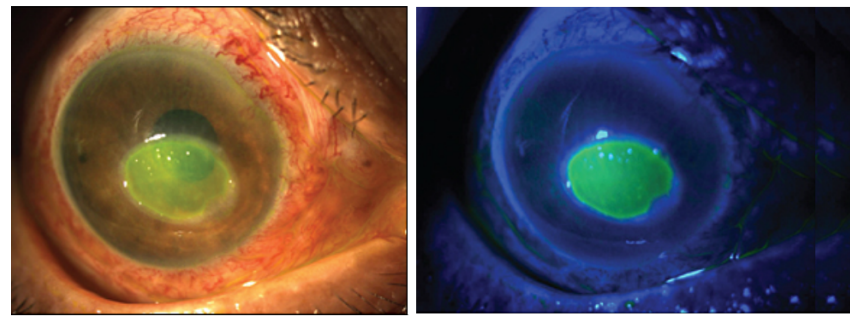

Figure 3: Corneal Abrasion. Note the fluorescein uptake where the corneal epithelial defect is present. This view is enhanced with cobalt blue lighting. Image courtesy of Kanski, JJ. Clinical ophthalmology: a systematic approach. Elsevier/Saunders; 2011

\section{Non-painful Red Eye}

A non-painful red eye with diffuse conjunctival injection may be caused by abnormalities of the eyelids. Debris and bacterial growth may be found in blepharitis and meibomitis which can spill over into the conjunctiva. Trichiasis (ingrowth of eyelashes) may cause mechanical irritation and malposition of the eyelid such as an ectropion and entropion.

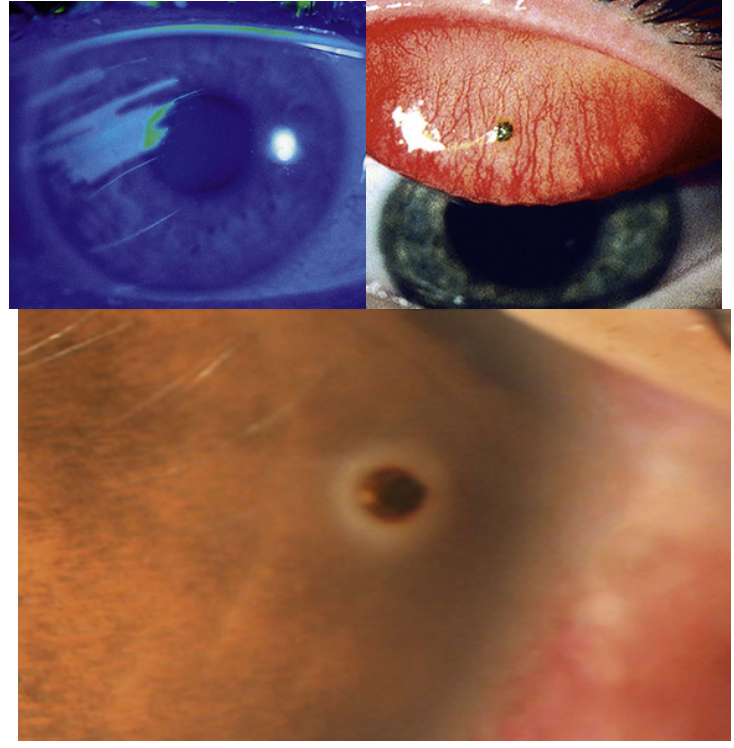

Figure 4: (a) Notice a metallic foreign body found by everting the upper eyelid. (b) shows how this same foreign body may result in a corneal abrasion. This pattern of abrasion should warrant eversion of the upper lid for inspection. (c) shows a high magnification photo of a corneal metallic foreign body. Image courtesy of Kanski, JJ. Clinical ophthalmology: a systematic approach. Elsevier/Saunders; 2011.

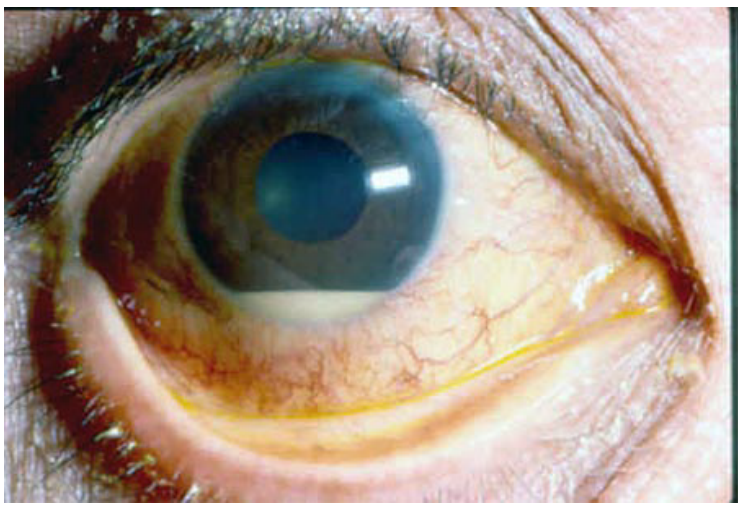

Figure 5: Hypopyon depicted as the white material, which is a leukocytic exudate, settling at the bottom of the anterior chamber. Image courtesy of Kanski, JJ. Clinical ophthalmology: a systematic approach. Elsevier/ Saunders; 2011.

In the absence of lid abnormalities, diffuse non-painful conjunctival injection may be caused by conjunctivitis. The distinctions between follicular and papillary conjunctivitis are helpful to determine treatment but these often require slit lamp examination. Follicles are best seen in the inferior conjunctival fornix, often called cul-de-sac. Papillae can be small or 'giant' and present a mosaic-like or studded cobblestone pattern. The most commonly encountered red eye may be viral conjunctivitis (Figure 8), colloquially called a pink eye. These patients may also endorse having a recent upper respiratory tract infection, sore throat, or contact with someone who was ill. They may also have cervical lymphadenopathy. Patients may describe their eyes as being matted shut when they wake up in the morning and mention that their eyes express a clear discharge. Viral conjunctivitis is very contagious and strict hand hygiene is to be followed to avoid outbreaks 
Citation: Braich PS, Kuriakose R (2016) How to Triage the Red Eye: Considerations for Non-ophthalmic Providers. Adv Practice Nurs 2:105. doi:10.4172/2573-0347.1000105

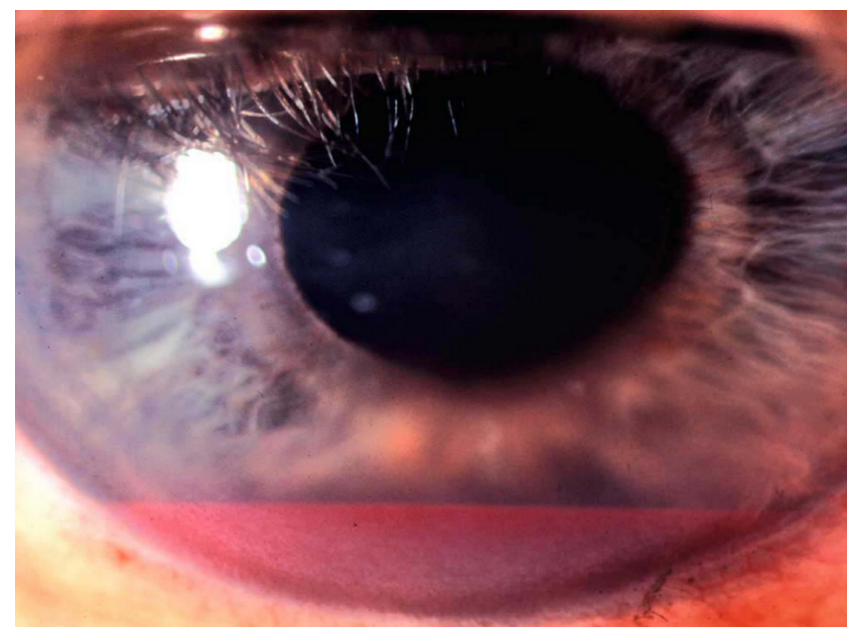

Figure 6: Hyphema depicted as blood and blood products settling at the bottom of the anterior chamber. Image courtesy of Kanski, JJ. Clinical ophthalmology: a systematic approach. Elsevier/Saunders; 2011.

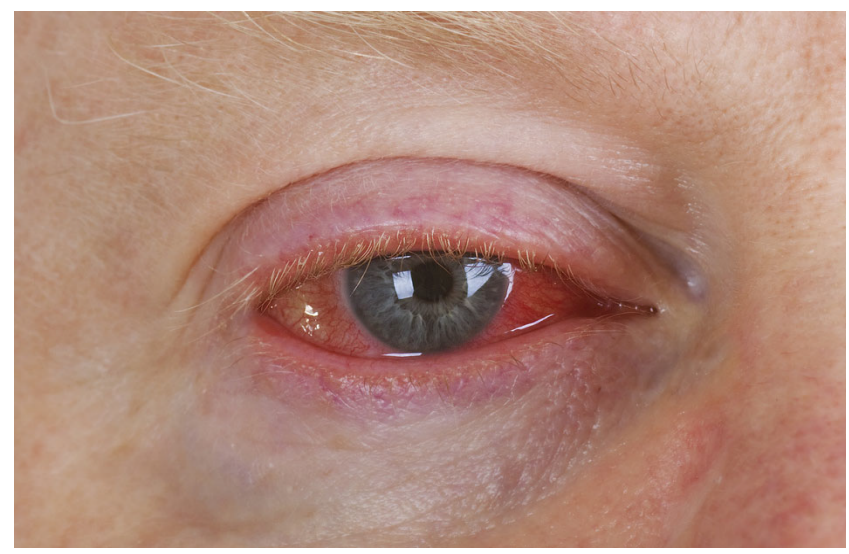

Figure 7: Viral conjunctivitis also colloquially called pink eye. Close up the conjunctiva may have a boggy appearance. The inferior fornix may reveal follicles. Image courtesy of Kanski, JJ. Clinical ophthalmology: a systematic approach. Elsevier/Saunders; 2011.

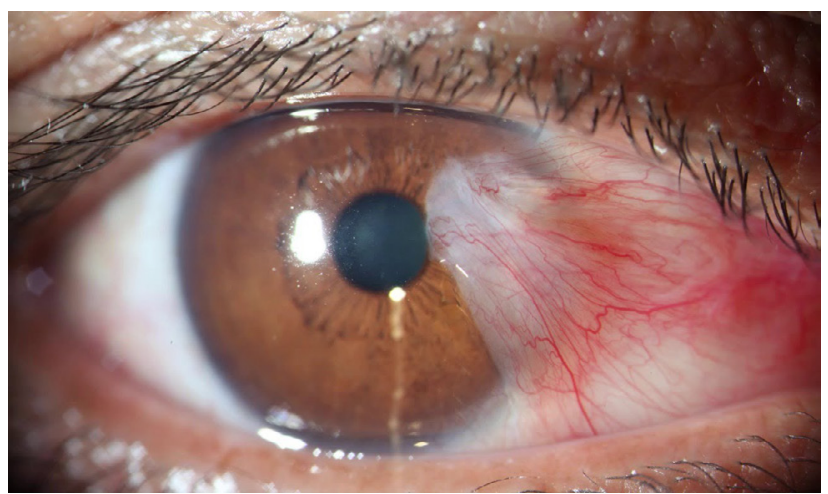

Figure 8: Pterygium. Notice the wing shaped fibrous tissue from the nasal aspect of the globe with overgrowth onto the cornea. Image courtesy of Kanski, JJ. Clinical ophthalmology: a systematic approach. Elsevier/ Saunders; 2011.

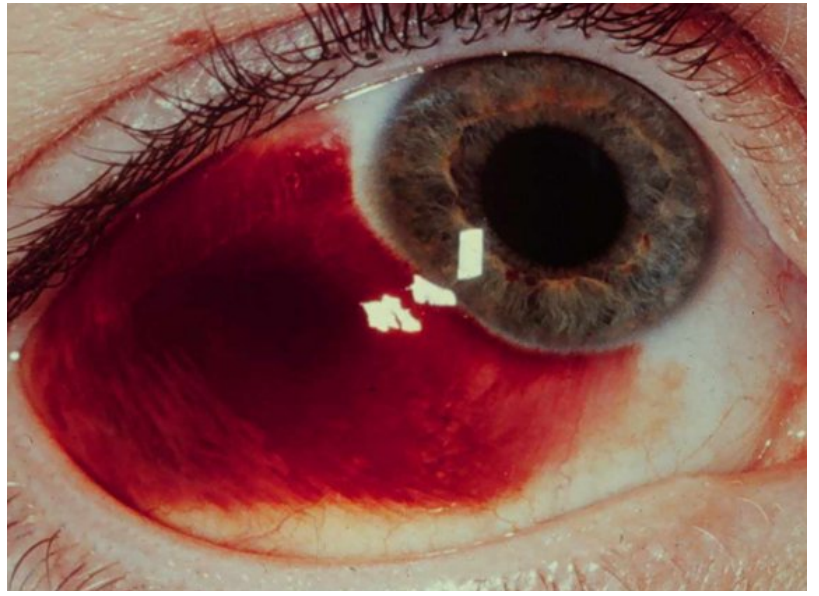

Figure 9: Subconjunctival hemorrhage. Image courtesy of Kanski, JJ. Clinical ophthalmology: a systematic approach. Elsevier/Saunders; 2011.

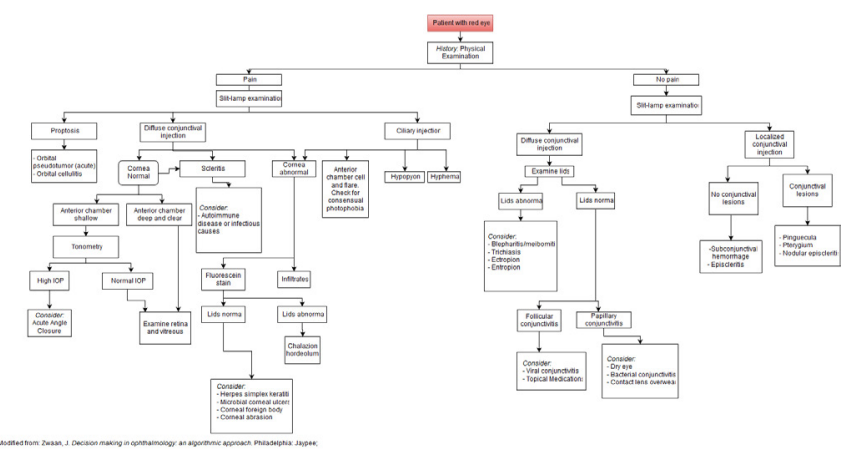

Figure 10: Flow chart for the rudimentary decision tree on triaging a painful red eye and non-painful red eyes. Image courtesy of Kanski, JJ. Clinical ophthalmology: a systematic approach. Elsevier/Saunders; 2011.

particularly at health care centers. The clinic supervisor should be notified so that your sanitization protocol can be followed when these patients are encountered.

Non-painful focal lesions of the conjunctiva may become irritated and inflamed. Pinguecula and pterygium are easily diagnosed (Figure 9). These lesions may arise from the nasal or the temporal aspect of the eye. Atypical inflamed lesions may need to be excised to rule out malignancy. Localized non-painful 'redness' of the conjunctiva may represents episcleritis, vascular injection (many etiologies), or a subconjunctival hemorrhage (Figure 10), which is a localized collection of blood beneath the conjunctiva with normal conjunctival vessels. Both conditions are benign, but occasionally, the first may require the use of topical nonsteroidal drugs. Subconjunctival hemorrhage can occur secondarily from trauma or from Valsalva maneuvers. They end up worrying the patient who is otherwise asymptomatic. Patients should be reassured about their condition resolving on its own in a few weeks. Recurrent subconjunctival hemorrhage without trauma warrants a hematologic workup.

Rarely, a non-painful red eye may suggest a malignancy or cerebral vascular abnormalities so speaking with your ophthalmic provider is encouraged.

This brief communications is in no way a substitute for a formal ophthalmic consultation and is only meant to be a cursory review of 
Citation: Braich PS, Kuriakose R (2016) How to Triage the Red Eye: Considerations for Non-ophthalmic Providers. Adv Practice Nurs 2:105. doi:10.4172/2573-0347.1000105

Page 4 of 4

how to triage the red eye. For a detailed reading, please refer to formal ophthalmology textbooks. For emergency room settings a useful reference the Wills Eye Manual [3].

\section{References}

1. Kanski JJ, Bowling B (2011) Clinical ophthalmology: a systematic approach (7th edn.), Edinburgh: Elsevier/Saunders.
2. Zwaan J (2014) Decision making in ophthalmology: an algorithmic approach Philadelphia: Jaypee.

3. Gerstenblith AT, Rabinowitz M (2012) The Wills eye manual: office and emergency room diagnosis and treatment of eye disease (6th edn.), Philadelphia: Wolters Kluwer/Lippincott Williams \& Wilkins. 